\title{
Bilateral occlusion of basilar artery branches
}

\author{
C. M I L L E R F ISHER
}

From the Neurology Service of the Massachusetts General Hospital and the Department of Neurology, Harvard Medical School, Boston, Massachusetts, USA

SUMMARY In a case in which the patient became totally paralysed except for blinking and vertical eye movements, microscopic serial sections of the pons showed bilateral infarcts which were due to occlusion of two small basilar branch arteries, one on each side. One basilar branch was occluded by an atheroma lying at its junction with the basilar artery and the other by an intramural dissection within the wall of the basilar artery. This case provides the clinicopathological correlation for two further basilar branch infarcts. An unusual finding was that one of the branch arteries supplied the basis pontis and medulla bilaterally.

The acute onset of total paralysis of the body with preservation of alertness, blinking, and vertical eye movements generally indicates brainstem ischaemia resulting from thrombosis of the basilar artery or the vertebral arteries. It was surprising, therefore, on pathological examination of such a case to find that, although the right vertebral artery was occluded, the basilar and left vertebral arteries were fully patent. Sections of the brainstem disclosed infarcts in the lower pons bilaterally which on microscopic examination proved to be the result of occlusion of two small arterial branches arising from the lower part of the basilar artery.

Investigation of the vascular anatomy in cases of basilar branch occlusion requires special preparation since routine horizontal sectioning of the brainstem results in disruption of small arterial branches of the basilar artery. This makes it impossible to trace in continuity the involved vessel from the infarct to its origin from the basilar artery and to identify the nature of the vascular obstruction. Passing a string beneath the basilar artery in order to suspend the brain in formalin is also contraindicated. We have used the technique of sectioning the brainstem frontally, beginning posteriorly in the region of the fourth ventricle and moving forward in thin sections until the infarct is found. The rest of the brainstem with attached vessels is then embedded as a block in paraffin and serially sectioned. In two previous cases (Fisher and Caplan, 1971) it was possible using this method to identify the site and nature of the occlusions. Since it is rarely feasible to com-

This study was supported in part by NINCDS, Grant NS05152. Accepted 16 August 1977 bine in one case a good neurological study and the necessary special neuropathological investigation, the present single case is reported here.

\section{Clinical details}

The patient, a mildly diabetic man aged 71 years, had severe hypertension for 15 to 20 years. Seven years before his final admission to hospital, he had successfully undergone surgery for a dissecting thoracic aneurysm. Two months before, he suffered a minor stroke which began with falling and swaying to the right, intermittent diplopia, and an inability to control his feet. Next day his right arm was numb and slightly weak, and the next day speech became dysarthric. On examination at that time there was weakness of the right side of the face, moderate dysarthria, slight weakness and a cerebellar-like ataxia or clumsiness of the right upper extremity, slight weakness of the right leg, brisker tendon reflexes on the right, a right $\mathrm{Bab}$ inski sign, and listing to the right on walking and on the Romberg test. The blood pressure was $200 / 100 \mathrm{mmHg}$. All abnormalities except slight dysarthria disappeared in about 10 days.

On the day of final admission, the patient vomited four times and noted dysarthria and weakness of both legs. During the next four hours there was tingling in the left arm and toes, choking on liquids and heavy sweating. He had been receiving methyldopa $250 \mathrm{mg}$ three times daily and triamterene $100 \mathrm{mg}$ daily. Examination showed weakness of the left side of the face, slight weakness of the left arm and leg, moderate dysarthria, and bilateral Babinski signs. On trying to walk he fell to the left. The blood pressure was 1182 
240/140 mmHg. Next day dysarthria was severe, the left arm was extremely weak, and the left leg did not move at all. The blood pressure was 190/ $130 \mathrm{mmHg}$. Hydralazine $35 \mathrm{mg}$ was given intravenously and four hours later the blood pressure had fallen to $120 / 80 \mathrm{mmHg}$. The left side was totally paralysed, and paralysis of conjugate lateral gaze to the right was noted. Atrial fibrillation appeared. Heparin intravenously was begun. Eight hours later, the blood pressure having remained about $140 / 80 \mathrm{mmHg}$, all limbs were paralysed, and the patient was mute. Horizontal eye movements were absent except for abduction of the left eye.
Secretions pooled in the throat. The patient opened and closed his eyes on command. Pinching either arm evoked an extensor posture. Both plantar responses were extensor. The blood pressure had risen to $170 / 80 \mathrm{mmHg}$. The patient died on the ninth day.

\section{Neuropathological findings}

Gross examination of the brain showed moderate atherosclerosis of the vessels at the base and occlusion of the right vertebral artery $5 \mathrm{~mm}$ from the basilar artery. The basilar artery was patent.
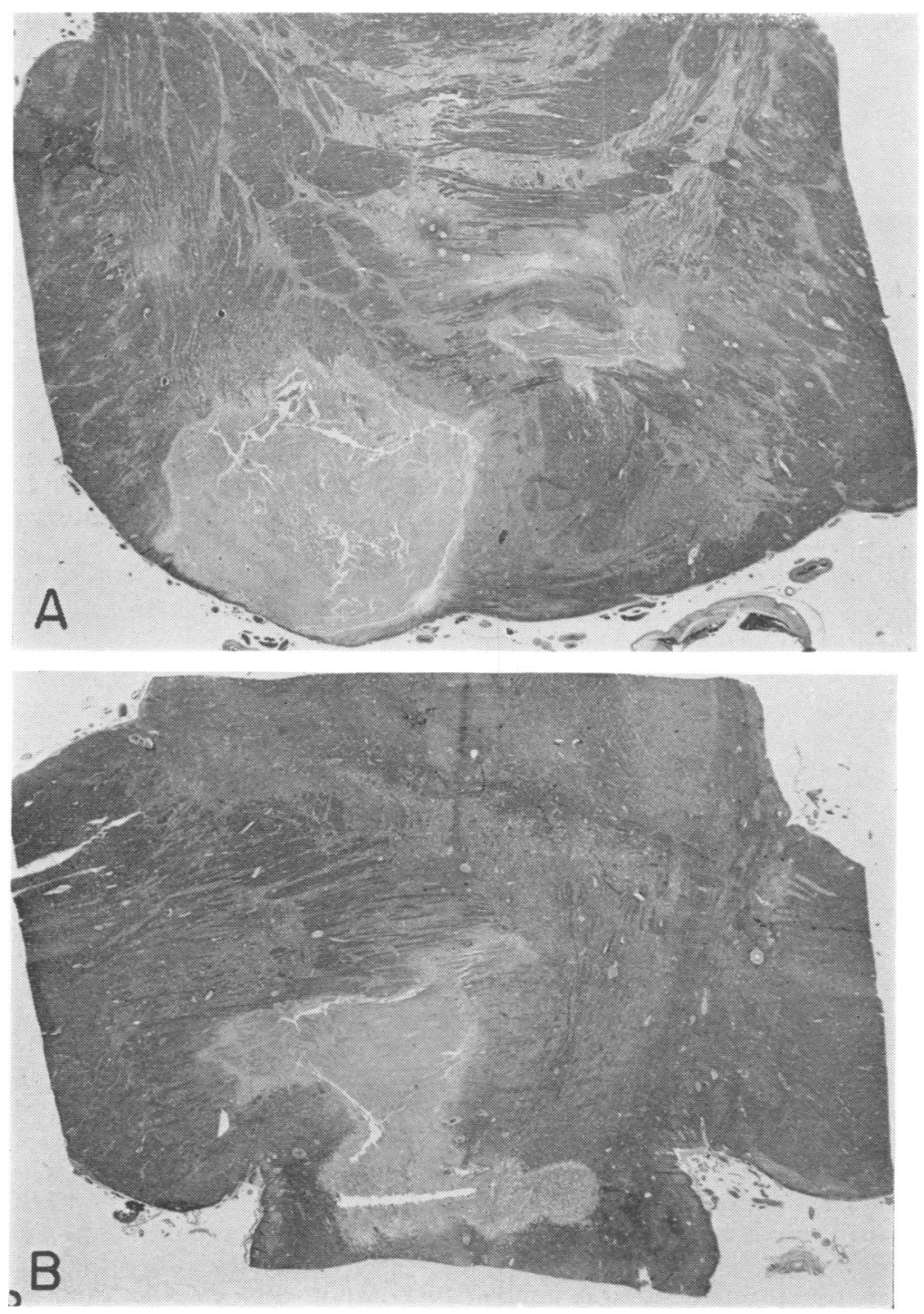

Fig. 1 Recent infarct on right side of pons. Superficial $(A)$, and deep (B). PTAH $\times 3 \frac{1}{2}$. 


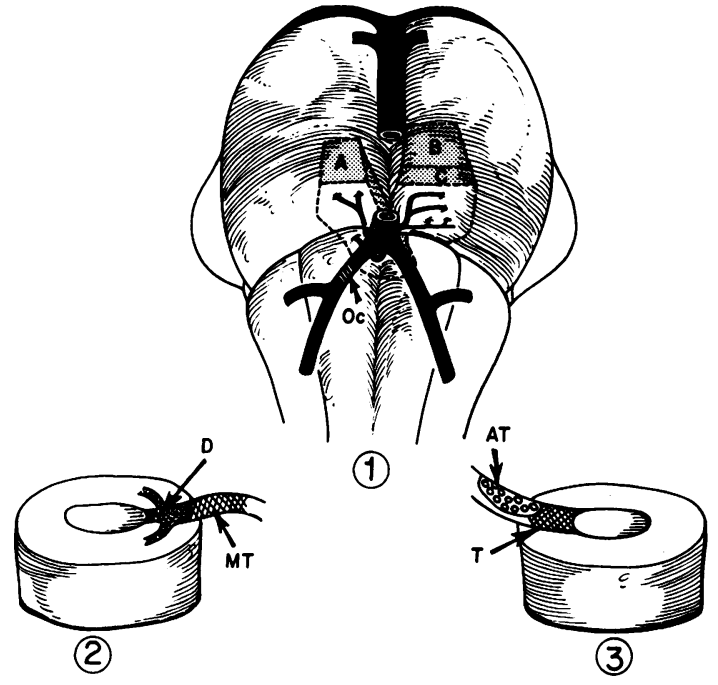

Fig. 2 Diagram of (1) location of infarcts and arrangement of vessels, and $(2,3)$ occlusions of basilar artery branches. $A=$ recent infarct on right side, $B=$ recent infarct on left, $C=$ older infarct on left, $O c=$ occlusion of right vertebral artery; (2) branch occlusion of right side, $D=$ dissection, $M T=$ mixture of fatty macrophages and thrombus; (3) branch occlusion of left side, $A T=$ plaque of atheroma, $T=$ thrombus.

The cerebral hemispheres contained three small lesions - two petechial haemorrhages, one in the right occipital cortex and one in the posterior parietal cortex, and a $6 \times 6 \mathrm{~mm}$ lacune in the left parietal white matter. On the assumption that the strokes were the result of small artery disease of the brainstem, the pons and upper medulla were sectioned frontally moving posteroanteriorly as already described until a softening appeared in the lower pons. The block was then embedded in paraffin and sectioned in a frontal plane. All sections were stained with phosphotungstic acid haematoxylin.

A small old infarct in the lower basis pontis on the left side probably accounted for the minor right sided stroke about two months before death. Recent extensive bilateral infarction of the basis pontis explained the final illness. This large recent softening consisted of two separate parts, one on each side of the lower pons. The recent infarct on the left side was in the same vascular territory as the old infarct of which it appeared to be an extension. This combined old and recent infarction resulted from occlusion of a small branch of the lower basilar artery. The recent infarct on the right side was due to occlusion of a small median branch of the basilar artery at its bifurcation. It was noteworthy that this infarct extended across the midline to involve several millimetres of tissue of the opposite side-that is, a single branch artery supplied a segment of the lower brainstem bilaterally.

\section{RECENT INFARCT ON RIGHT SIDE}

This infarct (Figs. 1A and 1B, and Fig. 2), which was in a premacrophage stage of evolution, lay in the lowermost pons and the adjacent upper 2.5 $\mathrm{mm}$ of the medulla oblongata. It measured approximately $11 \mathrm{~mm}$ transversely, $8 \mathrm{~mm}$ vertically, and $14 \mathrm{~mm}$ anteroposteriorly. It was irregular in shape and narrowed posteriorly. The downward coursing corticospinal fibres were interrupted mainly within the pons. The right medullary pyramid was extensively damaged while the left pyramid showed a $4 \times 3 \mathrm{~mm}$ softening (Fig. 1B) in addition to scattered smaller infarcts $1-2 \mathrm{~mm}$ in extent. Deep in the pons the infarct also crossed the midline by about $3 \mathrm{~mm}$.

The small arteries running within the infarcted tissue were traced proximally to their origin from a single small artery which coursed through the subarachnoid space to arise from the posterior aspect of the basilar artery in the midline (Fig. 2). Measuring about $500 \mu \mathrm{m}$ in diameter at its origin, the branch ran upwards for a few millimetres in the anterior median fissure of the medulla between the pyramids to enter the foramen caecum in whose depths it divided into a spray of some 15 or 20 small vessels which supplied all regions of the infarct bilaterally.

The arterial branch was occluded for a distance of one millimetre from its origin from the basilar artery by a mixture of fatty macrophages, red blood cells and thrombus that was undergoing fibroblastic organisation, a process that appeared to be older than a few days (Fig. 3A). Further distally the artery was normal and blood-containing. Where the branch traversed the wall of the basilar artery it entered a subintimal plaque of fibrous atheroma $2 \mathrm{~mm}$ thick. Here the artery appeared to be disrupted by a small mass of red blood cells that extended for 2 to $5 \mathrm{~mm}$ horizontally within the plaque, creating a crevice of local dissection $0.2 \mathrm{~mm}$ wide and approximately $0.5 \mathrm{~mm}$ vertically (Fig. 3B). The dissection which lay between the inner two-thirds and outer third of the plaque did not communicate with the lumen of the basilar artery. A small patent blood-containing channel which could be traced through the inner portion of the plaque probably was the remainder of the intramural course of the branch. It was difficult to interpret the occlusive process. 

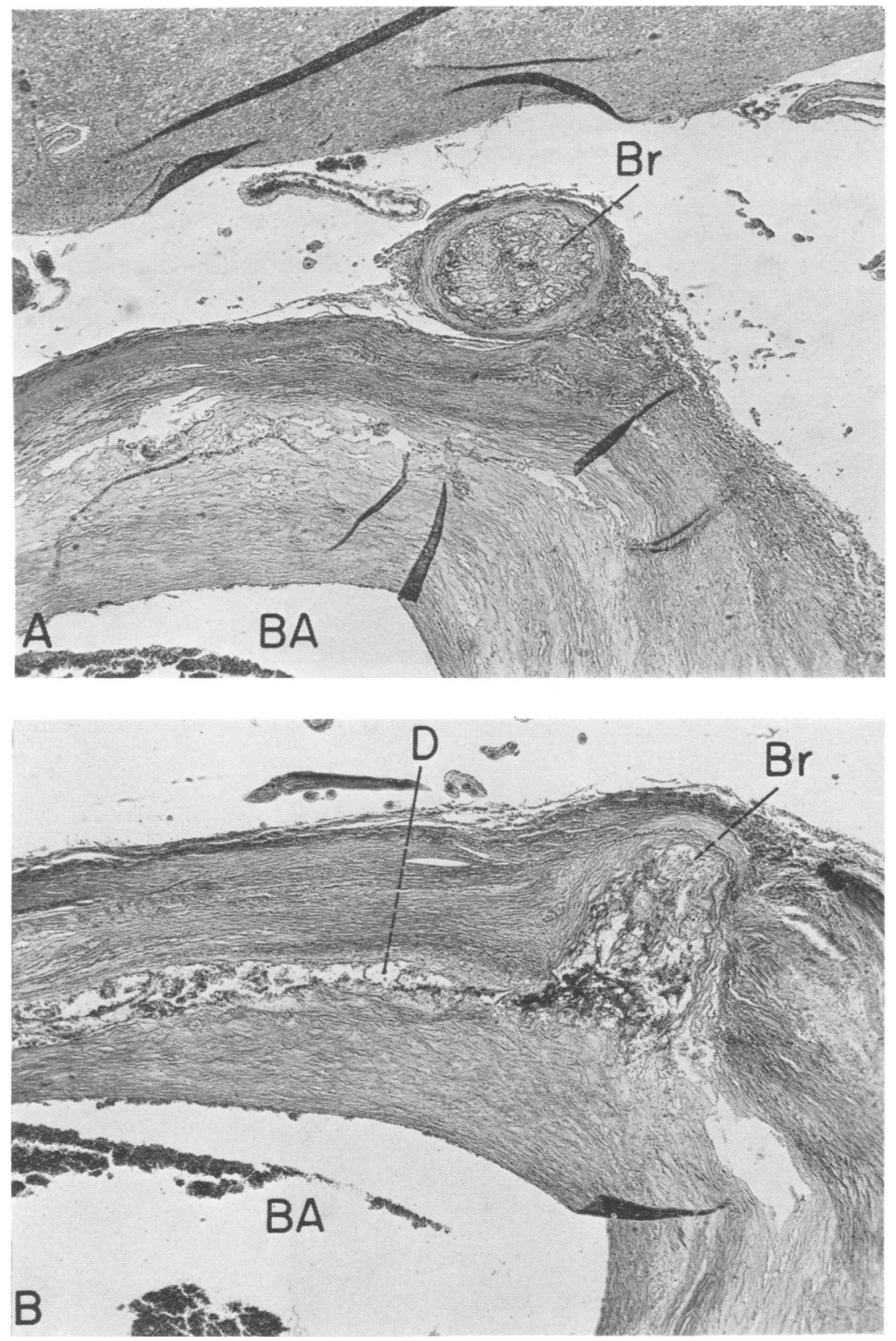

Fig. 3 Basilar branch corresponding to infarct in Fig. 1 and $A$ in Fig. 2, occluded near its origin $(A)$, and disrupted intramurally at site of dissection $(B)$. $B A=$ basilar artery, $B r=$ branch, $D=$ dissection. PT $A H \times 50$. 
One possibility was that the vessel had become severely narrowed by a lipid-macrophage atheroma into which a local haemorrhage had occurred producing the mixture described. Or possibly trauma had damaged the small branch in its intramural portion resulting in haemorrhage and local dissection. Microdissection of this type in the wall of the basilar artery has not been encountered previously.

Comment This recent infarct corresponded with the first part of the final stroke, namely, a severe left hemiplegia and right lateral gaze palsy, preceded by dysarthria, vomiting, sweating, and tingling in the left arm and toes. These manifestations, except for weakness and dysarthria, reflect penetration of the ischaemia into the tegmentum of the pontomedullary border zone. Profuse sweating is a not uncommon feature of pontine ischaemia. The report of weakness of both legs at the onset could be the result of the bilateral pyramidal lesions. When the final stroke approached its height, the only horizontal eye movement was abduction of the left eye, a state which has been reported previously as a transient occurrence associated with a lacunar infarct in the lower pons (Fisher, 1967).

The pathological findings were of special interest in that a single basilar branch supplied the brainstem bilaterally although predominantly on one side. This is probably the first time this pattern of
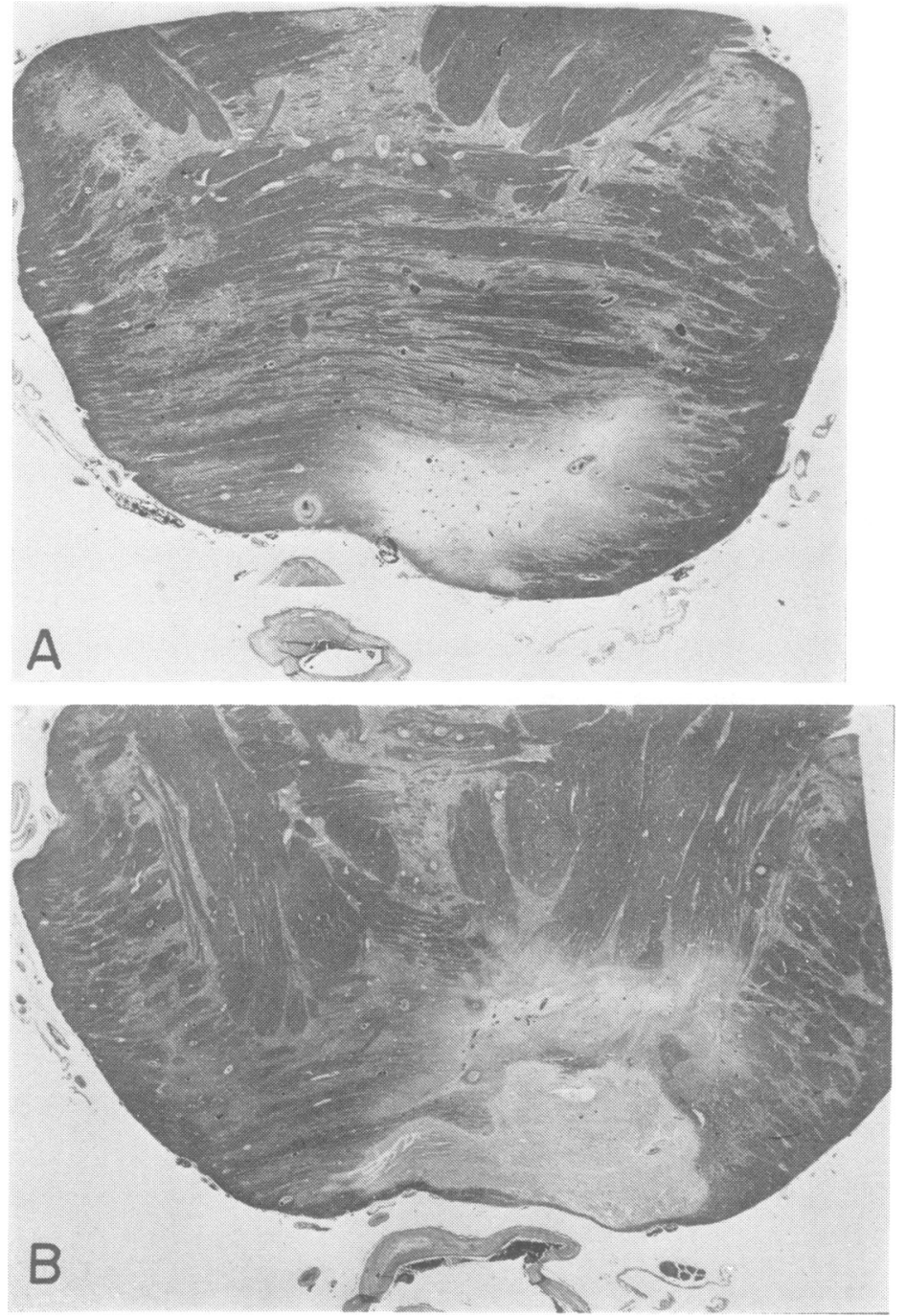

Fig. $4 A$ shows old infarct on left side, $B$ recent infarct on left side. $A$ is superficial, $B$ is deep. PT $A H \times 3 \frac{1}{2}$. 
blood supply has been demonstrated and may explain unexpected bilateral brainstem signs when the arteries are angiographically patent or only one vertebral artery is occluded.

SMALL OLD INFARCT ON LEFT SIDE

This infarct which showed numerous large macrophages and fine fibrillar connective tissue was several weeks old and correlated best with the slight right sided stroke. It lay in the lowermost pons on the left side (Fig. 4A) and measured $7 \mathrm{~mm}$ transversely, $3 \mathrm{~mm}$ vertically and 3 to $6 \mathrm{~mm}$ anteroposteriorly, extending further posteriorly in its superior portion. The vessels supplying the territory of this infarct were readily traced to a small artery approximately $300 \mu \mathrm{m}$ in diameter which ran in the subarachnoid space to join another artery of about the same size about $5 \mathrm{~mm}$ before the resulting common trunk entered the lateral aspect of the basilar artery about $3 \mathrm{~mm}$ above the lower bifurcation (Fig. 2). The 'other artery' was the artery which supplied the territory of the recent left sided infarct to be described next.

Comment The clinical manifestations associated with this old softening in the lower pons included slight weakness of the face, arm, and leg on the right side, moderate dysarthria, transient diplopia, listing to the right, numbness of the right arm, cerebellar-like ataxia of the right upper extremity, and increased tendon reflexes and a Babinski sign on the right side. The mechanism by which power on the right side of the body is diffusely but only slightly reduced is not readily explained, but a limited ischaemia of the entire cross-section of the corticospinal system may be the answer. The combination of ipsilateral hemiparesis and ataxia is also of obscure origin although recognised as a sign at times of unilateral involvement of the basis pontis. Diplopia and numbness are indicative of tegmental involvement. It is of interest that the neurological deficit disappeared despite the permanent anatomical damage.

The infarct accurately conformed to the vascular territory of one of two divisions of a basilar branch artery which itself was occluded. This arrangement suggests a local embolus to the patent branch from the parent artery but the stroke apparently evolved in two days, unlike embolism.

LARGE RECENT INFARCT ON LEFT SIDE

As already mentioned, this infarct was in the same region as the small old infarct described above, which it more or less surrounded and extended. It lay in the lowermost basis pontis anteriorly and medially, measuring $9 \mathrm{~mm}$ transversely, $6 \mathrm{~mm}$ vertically, and $10 \mathrm{~mm}$ anteroposteriorly (Fig. 4B). It did not cross the midline. Its vessel of supply which penetrated the pons close to the midline was traced back to its junction with the branch supplying the other part of the small old infarct.

The common trunk which measured about 500 $\mu \mathrm{m}$ in diameter was traced through the subarachnoid space to its origin from the basilar artery. At this point and extending intramurally for a short distance, the branch was almost obliterated by a pure lipid atheroma $1 \mathrm{~mm}$ in length deposited upon which was a fibrin micro-thrombus which occluded the remaining lumen-a typical 'junctional' occlusion (Figs. 5A, B).

Comment This infarct obviously accounted for the final right sided hemiplegia which when added to the left hemiplegia resulted in complete paralysis of the body.

The vascular lesion consisted of a bead of atheroma with a superimposed micro-thrombus. Both old and recent infarcts lay in the territory of supply of the basilar branch and the presence of infarcts of different ages in the two divisions of the branch is puzzling. As already mentioned, local embolism may have occurred. Another possibility is that complete occlusion of the branch was precipitated by excessive lowering of the patient's blood pressure; or collateral flow compensated for the occlusion until the blood pressure fell abruptly. No subarachnoid anastomotic connections were present, and any collateral flow would have to have been at the capillary level.

\section{Discussion}

The principal points for discussion have been included in the comments above and only a few further observations remain to be made.

It should be emphasised that the occlusion of the right vertebral artery was more than $5 \mathrm{~mm}$ removed from the involved basilar branches and, therefore, in all probability could not have been a factor in the infarcts.

The clinical picture in this case was one which would ordinarily have conjured up the diagnosis of a massive pontine lesion, especially of the basis pontis. That two strategically placed basilar branch infarcts could evoke a similar catastrophic paralysis is an unusual occurrence which must be added to diagnostic considerations.

The clinicopathological findings are of special interest in that the manifestations of two further brainstem or lacunar infarcts are provided to add to the two previously described (Fisher and 

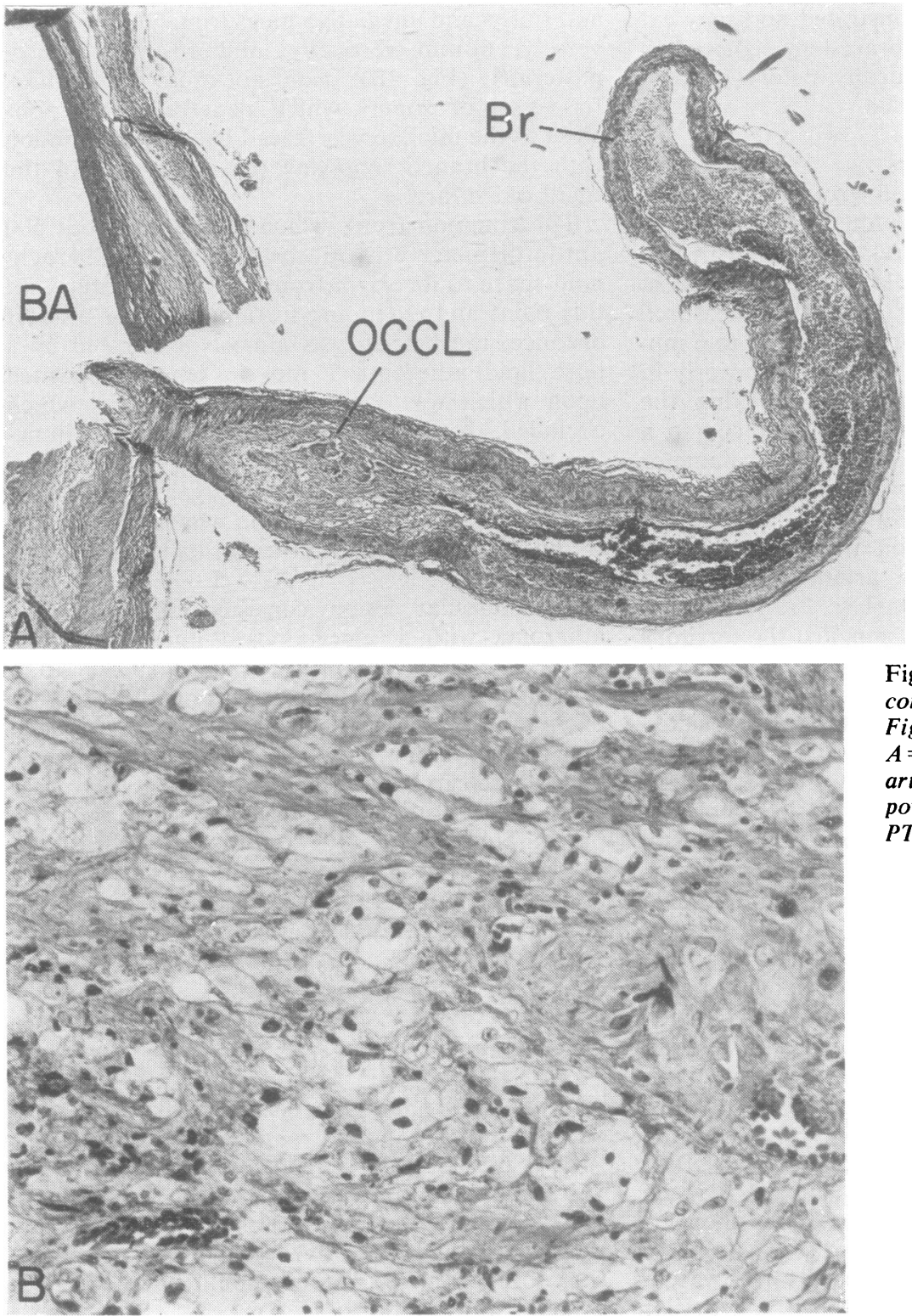

Fig. 5 Basilar branch artery corresponding to infarct in Fig. 4, and B and C Fig. 2. $A=o c c l u s i o n$ near basilar artery. PTAH $\times 45 ; B=$ high power view of atheroma. PTAH $\times 290$.

Caplan, 1971). The main features have already been commented on and further discussion is unnecessary.

The two vascular lesions exemplify the two main types of branch occlusion described thus far (Fisher and Caplan, 1971), one an occluding atheroma at the junction of branch and basilar artery, and the other an interruption of the branch within the wall of the basilar artery at the site of an atherosclerotic basilar plaque. This is the first time that a dissection in the basilar wall was a factor, apparently compressing the branch as it traversed the wall. The beads of atheroma which are deposited in basilar branch arteries in the presence of hypertension consist almost entirely of foamy, fatty macrophages, an appearance which emphasises for the pathologist the fundamental role of lipid in atherosclerosis.

It is worth mentioning again that in this case it was demonstrated that a single paramedian pene- 
trating branch of the basilar artery straddled the midline to supply central regions of the basis pontis and medulla bilaterally. It occasionally happens that in a minor brainstem stroke with bilateral manifestations, angiography which does not opacify small-calibre penetrating branches fails to show a vascular lesion and it may be suspected on the present evidence that a branch occlusion is responsible. In the present case, one of the branch vessels entered the 'foramen caecum', a portal of entry which allows small arteries to reach the brainstem tegmentum directly without first penetrating the basis pontis or pyramids. Adequate clinicopathological studies in such cases are lacking.

Finally, the hazards of precipitously lowering the blood pressure during the acute stage of a thrombotic stroke may be illustrated in this case not only by the disastrous clinical course but also by the pathological finding of an old and a recent infarct in the territory of a single basilar artery branch. It is our practice to avoid hypotensive agents in ischaemic brain disease and allow the blood pressure to settle naturally.

\section{References}

Fisher, C. M. (1967). Some neuro-ophthalmological observations. Journal of Neurology, Neurosurgery, and Psychiatry, 30, 383-392.

Fisher, C. M., and Caplan, L. R. (1971). Basilar artery branch occlusion: a cause of pontine infarction. Neurology (Minneapolis), 21, 900-905. 